\title{
A NEW PROCEDURE FOR SOLVING THE MULTI-ITEM, SINGLE-LEVEL, LIMITED CAPACITY, LOT-SIZING AND SCHEDULING PROBLEM WITH SEQUENCE-DEPENDENT SET-UP COSTS
}

\section{Tore Grünert, RWTH Aachen}

We consider the problem of determining lot-sizes and a production schedule for different products which are produced on the same assembly line. It is required that the demand for each product in each period and the corresponding production times are known. The objective is to minimize the sum of sequencedependent set-up costs and inventory costs. The problem belongs to the class NP, for which no optimal solution procedures exist that solve realistic-size problems.

Many heuristic procedures solve relaxations of the described problem, for instance the Dixon/Silver and Günther heuristic for the DLSP, the Lagrange-relaxation algorithm by Fleischmann for the CLSP.

Our algorithm is based on a combination of tabu search, a meta-heuristic developed by Glover, and Dijkstra's shortest path algorithm. We demonstrate how the implemented swapping techniques and the problem-specific modelling affect the computational effectiveness. Possible extensions of the basic algorithm will also be considered. 